\title{
Nanostructure Evolution in Poly(propylene) During Mechanical Testing
}

\author{
Norbert Stribeck,* Ulrich Nöchel, Sérgio S. Funari, Tom Schubert, \\ Andreas Timmann
}

Slow continuous mechanical tests of oriented poly(propylene) (PP) are monitored by combined two-dimensional (2D) ultra-small-angle X-ray scattering (USAXS) and small-angle X-ray scattering (SAXS). Patterns are transformed to the 3D chord distribution (CDF), a real-space representation of nanostructure. From the CDF, topological parameters of the semicrystalline domain structure are extracted and discussed. A continuous-strain test shows fracture and release of weak lamellae (2-10\% strain). Beyond that we see conversion of lamellae into needles. As all layers are consumed the material breaks. Fatigue is studied in load-reversal (between 10 and 35\% strain). We report cyclic (damped) crystallization, layer break, and relaxation melting.

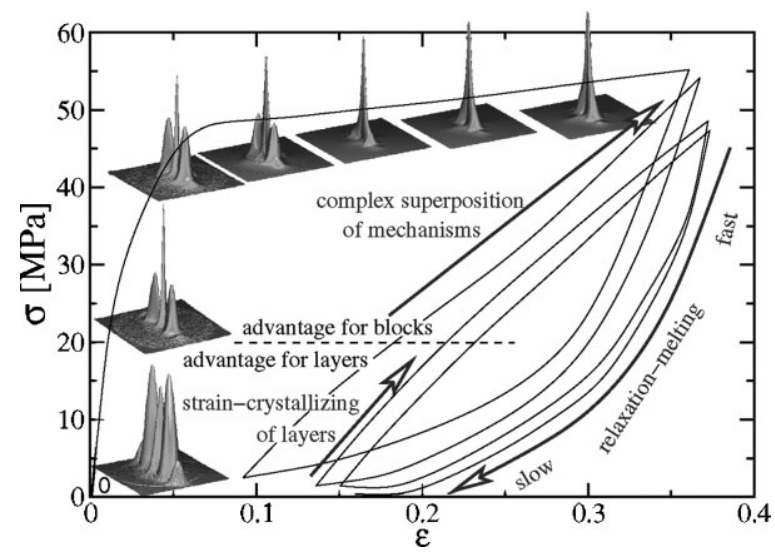

\section{Introduction}

The understanding of failure or fatigue mechanisms in polymer materials is of practical relevance, because new light-weight materials with special toughness or low fatigue are urgently sought after. An example is the automotive industry, which needs such materials in order to accomplish the goal of fuel reduction by the reduction of weight.

These mechanisms can be studied by methods which provide time-resolved in situ data, like the small-angle Xray scattering (SAXS). Nevertheless, the available instrumentation is enforcing a compromise. Either low-noise patterns are recorded with poor time resolution, or noisy patterns with good time-resolution are obtained. For phenomenological surveys it is suitable to relinquish the

N. Stribeck, U. Nöchel

Department of Chemistry, Institute of Technical and Macromolecular Chemistry, University of Hamburg, Bundesstr. 45, 20146 Hamburg, Germany

Fax: (+49) 4042838 6008; E-mail: norbert.stribeck@desy.de S. S. Funari, T. Schubert, A. Timmann

HASYLAB at DESY, Notkestr. 85, 22603 Hamburg, Germany postulate of recording low-noise scattering patterns using instruments equipped with a very fast detector ${ }^{[1]}$. In such an environment, structure evolution can be studied at strain rates of $\dot{\varepsilon} \approx 10^{-1} \mathrm{~s}^{-1}$, which are common in industrial processes. A selection of corresponding studies ${ }^{[2-5]}$ demonstrates both power and limits of this approach.

Whenever scattering patterns from anisotropic materials shall be evaluated quantitatively, the stretch-hold technique ${ }^{[6]}$ is frequently employed. ${ }^{[7-27]}$ Characteristic of this technique is stepwise elongation and recording of the scattering while the extensometer is idle. As instrumentation technique is advancing, it is now becoming possible to set up a truly dynamic experiment in which low-noise patterns are recorded with a sufficient frame rate, if only the strain rates are kept low $\left(\dot{\varepsilon} \approx 10^{-3} \mathrm{~s}^{-1}\right)$. To the best of our knowledge, the first paper on polymer deformation based on low-noise patterns with an exposure of $15 \mathrm{~s}$ and a cycle time of $30 \mathrm{~s}$ has been published by Chen et al. ${ }^{[28]} \mathrm{In}$ this manner, a stream of high-precision scattering data is obtained. Moreover, this stream can be considered quasicontinuous, if the variation of nanostructure from frame to frame is small. In this case, a survey of the mechanisms of structure evolution is becoming possible. 
One method of data evaluation starts from nanostructure models. The corresponding scattering patterns are computed and compared to the measured patterns, as has been demonstrated after a continuous-straining experiment of toughened ultra-high molecular-weight polyethylene. ${ }^{[28]}$ Another study ${ }^{[29]}$ presents SAXS data from a fatigue test. An isotropic polyurethane elastomer is subjected to load-reversal cycling, and the shape of the observed ellipsoidal reflection is compared to the model scattering of affinely deformed macrolattices (cf. chapter 10 in Stribeck $\left.{ }^{[30]}\right)$. On the other hand, instead of computing a model scattering pattern, it is possible to transform the measured complete $\mathrm{e}^{[30,31]}$ scattering patterns into a representation of the nanostructure in real space, ${ }^{[32]}$ where structure parameters can be extracted. In this article, the last-mentioned method is demonstrated for poly(propylene) (PP) both after a continuous-straining experiment, and after a fatigue test. We study highly oriented, hardelastic ${ }^{[33-37]}$ material, and strain in the normal direction of the crystalline lamellae. Thus, shear and the formation of micropores $^{[38,39]}$ are prevented ${ }^{[40]}$ for the most part.

Ultimately, we are pursuing this and similar studies in order to identify the mechanisms of nanostructure evolution which are controlling the failure or the fatigue of polymer materials. Such information is required, if a realistic computer model of a polymer material shall be set up in order to predict tailored materials properties by simulation of the models response to load as a function of structure. We admit that presently only experimental indications on the complexity of the mechanisms and the ranges of their activity can be reported. Nevertheless, such results appear to be an advance, compared to the predominantly homogeneous models ${ }^{[41]}$ which are commonly used to predict the properties of polymers.

The results presented in this paper are based on newly developed techniques, which have been presented and compared elsewhere. Because PP can show discrete smallangle scattering over a wide range of scattering angles, scattering patterns have been combined. An own article describes this technique which is based on desmearing and merging. ${ }^{[42]}$ Moreover, the earlier stretch-hold technique results in distinctly different results when applied to PP. The reason is a considerable relaxation of nanostructure, which starts immediately after stopping the extensometer (cf. earlier work ${ }^{[43]}$ and Section "Structure Relaxation after the Load-Reversal Test").

\section{Experimental Part}

\section{Material}

Commercial hard-elastic ${ }^{[33,44]}$ PP film (CelGard-PP ${ }^{\circledR}$, Lot \#884, as extruded by Hoechst-Celanese) of $25 \mu \mathrm{m}$ thickness was studied. The films were produced ${ }^{[34]}$ from the isotactic PP grade Hercules
Profax 6301 with a melt index $15.0\left(230^{\circ} \mathrm{C}\right)$ ASTM-D-1238. The weight-average molecular mass of the grade was $\bar{M}_{\mathrm{n}}=$ $128000 \mathrm{~g} \cdot \mathrm{L}^{-1}$. The polydispersity of the material was $\bar{M}_{\mathrm{w}} / \bar{M}_{\mathrm{n}}=4$. Test "bars" conforming to DIN 53504 were punched from the double-layer film resulting in samples of $50 \mu \mathrm{m}$ thickness. For this purpose a toggle-lever press manufactured by Zwick GmbH, Ulm, Germany was used. The axis of the test bar was oriented parallel to the principal axis of the uniaxially oriented films. Fiducial marks were applied by a rubber stamp with a line grid of $2 \mathrm{~mm}$ distance.

\section{Laboratory Tensile Tests}

Cross-section change of the central part of the test bar during mechanical tests was studied before the synchrotron experiments in the laboratory by a commercial extensometer (Zwicki Z1.0/ TH1S, Zwick GmbH, Ulm, Germany).

\section{Small-Angle X-Ray Scattering Environment}

Small-angle X-ray scattering was performed at the synchrotron beamline A2 at HASYLAB, Hamburg, Germany. The wavelength of the X-ray beam was $0.15 \mathrm{~nm}$, and the sample-detector distance was $3045 \mathrm{~mm}$. Scattering patterns were collected by a twodimensional (2D) position sensitive marccd 165 detector (mar research, Norderstedt, Germany) operated in $2048 \times 2048$ pixel mode (pixel size: $79 \times 79 \mu \mathrm{m}^{2}$ ).

\section{Ultra-Small Angle X-Ray Scattering (USAXS) Environment}

Ultra-small-angle X-ray scattering was performed at the synchrotron beamline BW4 at HASYLAB, Hamburg, Germany. The wavelength of the X-ray beam was $0.13 \mathrm{~nm}$, and the sampledetector distance was $8906 \mathrm{~mm}$. Detection and registration of the scattering patterns was the same as in the SAXS environment.

\section{Tensile Test at the Beamlines}

Tensile testing at the beamlines was performed with a self-made extensometer fit for application at HASYLAB beamlines, which had been built by one of us (T. S.). The machine performed symmetric drawing. Signals from load cell and transducer were recorded during the experiment. The sample was monitored (cf. Figure 1a) by a TV-camera, and video frames were grabbed and stored together with the experimental data. The experiment control software permitted to define eight intervals of different cross-head movements. In all experiments, the machine was operated at velocities of either $2 \mathrm{~mm} \cdot \mathrm{min}^{-1}$ (straining) or $-2 \mathrm{~mm} \cdot \mathrm{min}^{-1}$ (relaxation), respectively (cf. Figure $1 \mathrm{~d}$ ).

The true elongation $\varepsilon=\left(\ell-\ell_{0}\right) / \ell_{0}$ was computed from the initial distance, $\ell_{0}$, of the two fiducial marks enclosing the point of beam-irradiation, and the respective actual distance, $\ell$ (cf. Figure 1a). Thus, all the reported elongations were not derived from the extensometer state, but from the evolution of the fiducial marks on the sample as monitored in a video. The determination was rather accurate $(\Delta \varepsilon \approx 0.005)$ because of several reasons: (i) the fiducial grid was transferred to the sample from a high-precision rubber stamp. (ii) The sample was monitored by a macrolens. (iii) The image was additionally zoomed on the TV-screen so that 


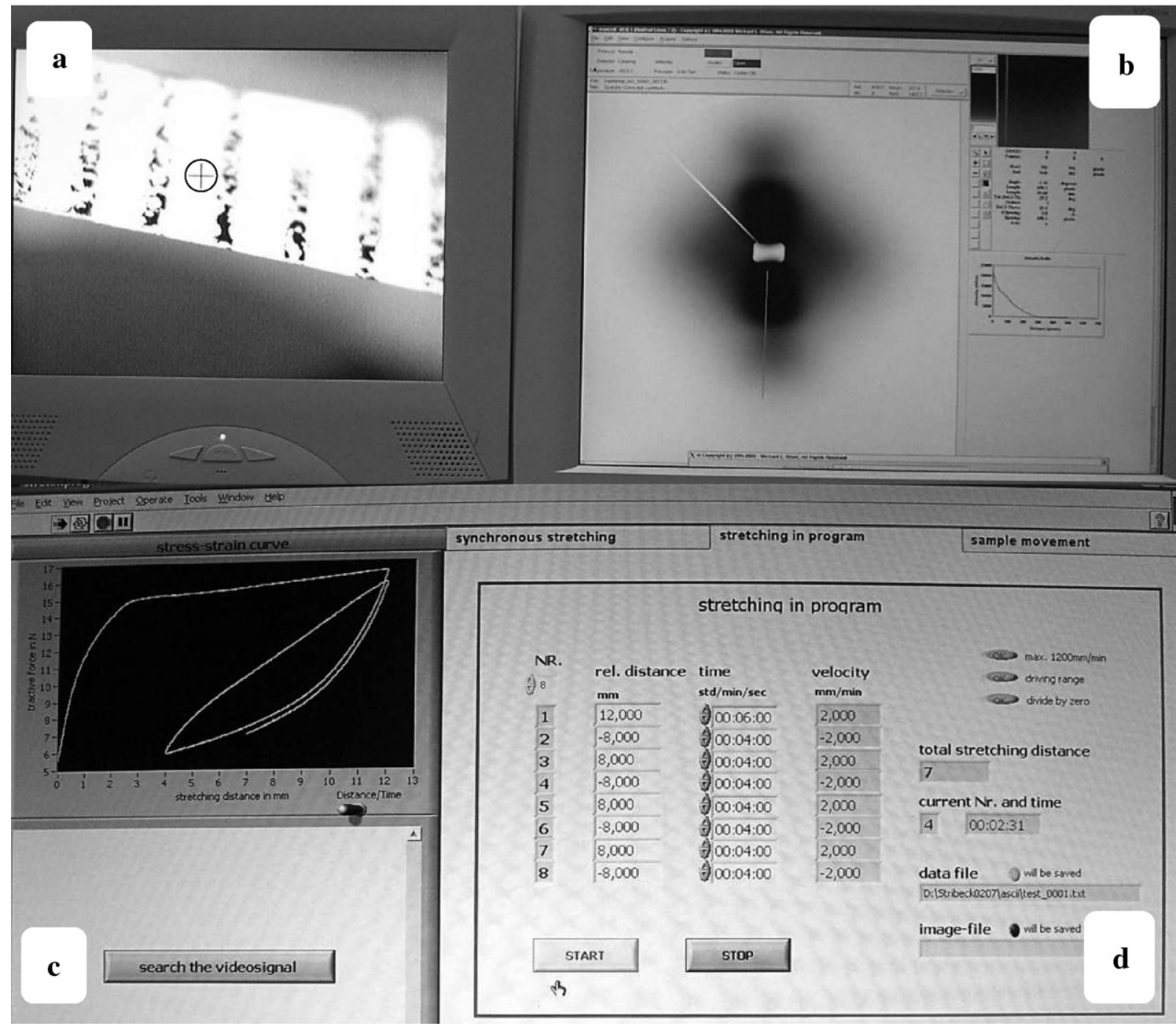

Figure 1. Mechanical testing of PP in progress at HASYLAB as watched on monitors. (a) Sample with fiducial marks. The area of X-ray irradiation marked by an encircled cross-hair. (b) Actual SAXS pattern. (c) Mechanical progress (force vs. machine strain). (d) Programming window.

the distances that must be measured were typically $10 \mathrm{~cm}$. During dynamic tensile testing between $\varepsilon=0$ and $\varepsilon=6$ the true strain rate, $\dot{\varepsilon}$, had been found to increase monotonously from $0.8 \times 10^{-3}$ to $1.1 \times 10^{-3} \mathrm{~s}^{-1}$

Scattering patterns were recorded every $60 \mathrm{~s}$ with an exposure of $49 \mathrm{~s}$. Progress was observed on monitors (Figure 1) outside the experimental hutch. One of the monitors showed the TV-image of the sample with the irradiation zone of the synchrotron beam indicated. For every SAXS pattern that was recorded, several video pictures were grabbed and stored together with the elapsed time. Other monitors displayed the actual SAXS pattern, the progress of the experiment reflected in the engineering stress-strain curve, and the progress of the extensometer program. Time, force, and the distance of the cross-heads were continuously recorded in an ASCII file. From this file the force can directly be associated to each scattering pattern. The true elongation was manually determined after the end of the experiment. For this purpose, the fiducial marks visible in the video pictures were consulted.

\section{Data Evaluation}

\section{Pattern Merging and Desmearing}

The applied evaluation of SAXS patterns requires the recording of low-noise images over the whole angular range where discrete scattering has been observed. Because the material shows both SAXS and USAXS, the SAXS has been desmeared and merged with the USAXS pattern as described in a previous article. ${ }^{[42]}$

\section{Chord-Distribution Function Analysis}

Our analysis starts from the scattering pattern and turns it into a representation of the nanostructure in real space. The only assumption is the presence of a multiphase 


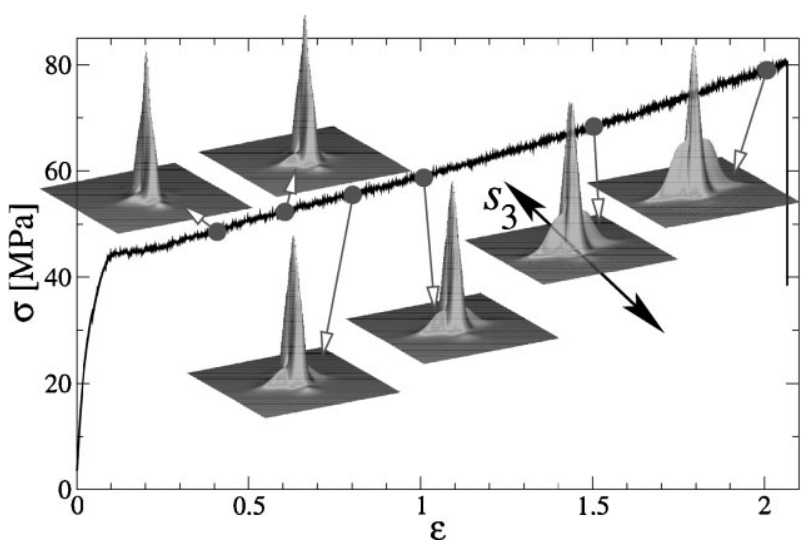

Figure 2. Hard-elastic PP. Continuous straining until break monitored by SAXS. The SAXS patterns show a region $-0.17 \mathrm{~nm}^{-1}$ $<s_{12}, s_{3}<0.17 \mathrm{~nm}^{-1}$. The bold arrow in one of the SAXS patterns indicates the straining direction $\left(s_{3}\right)$. Intensity on a logarithmic scale.

topology. The result is a multidimensional chord distribution function (CDF), $z(\mathbf{r}) \cdot{ }^{[32]}$ The method is exemplified in a textbook (Stribeck ${ }^{[30]}$, Section 8.5.5). For a schematic sketch of the steps of data analysis and the extraction of structural parameters from the CDF see, for example, Figure 2 in Stribeck et al. ${ }^{[45]}$ The method is extracting the topological information on nanostructure (e.g., a twophase topology, $\rho(\mathbf{r}) \in\left[\rho_{\text {cryst }}, \rho_{\text {amorph }}\right]$, of phases with distinct densities) from 2D SAXS patterns with uniaxial symmetry. The resulting CDF is an "edge-enhanced autocorrelation function" ${ }^{\text {[46-49] }}$ - the autocorrelation of the gradient field, $\nabla \rho(\mathbf{r})$. Thus, as a function of ghost displacement, $\mathbf{r}$, the multidimensional $\mathrm{CDF} z(\mathbf{r})$ shows peaks wherever there are domain surface contacts between domains in $\rho(\mathbf{r})$ and in its displaced ghost. The CDF with fiber symmetry in real space, $z\left(r_{12}, r_{3}\right)$, is computed from the fiber-symmetrical SAXS pattern, $I\left(s_{12}, s_{3}\right)$, of multiphase materials. ${ }^{[32]} \mathbf{s}=\left(s_{12}, s_{3}\right)$ is the scattering vector with its modulus defined by $|\mathbf{s}|=$ $s=(2 / \lambda) \sin \theta$. Here, $\lambda$ is the wavelength of radiation and $2 \theta$ is the scattering angle. In the historical context, the CDF is an extension of Ruland's interface distribution function $(\mathrm{IDF})^{[50]}$ to the multidimensional case or, in a different view, the Laplacian of Vonk's multidimensional correlation function. ${ }^{[51]}$

\section{Results and Discussion}

\section{Straining Until Break}

In the classical mechanical test the material has been extended until rupture. As the test is continuously monitored by respectively.
SAXS (Figure 2), no remarkable changes are found in the long linear part of the stress-strain curve. With increase in elongation, the higher orders of the long period vanish. All in all, the scattering of the material strongly decreases (in the leftmost pattern the intensity is ten-fold the intensity of the rightmost pattern). During the test the relative strength of an equatorial streak increases. If the patterns are interpreted directly, the growth of the distance between neighboring crystalline lamellae (long period) can be compared to the macroscopic elongation. The practical value of such results for the understanding of the nanomechanics is considered limited. ${ }^{[31]}$

Considerable changes which appear interesting for the application are observed in the interval of low elongations $(0<\varepsilon<0.4)$, which is again observed in the load-cycling experiment. The corresponding results are presented in the respective Section "Load-Cycling".

Moreover, the data of the continuous-strain experiment enable us to study the nanostructure evolution of hardelastic PP in the vicinity of rupture under dynamical conditions for the first time as compared to the stretchhold technique. ${ }^{[6,43]}$ As already shown by the scattering patterns (Figure 2), the scattering of the ensemble made from crystalline and amorphous lamellae continuously decreases while the equatorial scattering of a fibrillar system becomes more obvious. Whether or not there are still uncorrelated lamellae present at break, is difficult to tell from the scattering patterns directly. Therefore, we compute the multidimensional $\mathrm{CDF}^{[32]}$ and monitor the nanostructure evolution in physical space. Figure 3 shows the result. Already at an elongation $\varepsilon=1$, the influence of the nascent equatorial streak from the SAXS pattern is present in the CDF. Across the strong layer reflections two sharp ridges are running close to each other in the direction parallel to the meridian $\left(r_{3}\right)$. Because the distance between these ridges is short, the distance between opposite surfaces of the corresponding domains is short. This means that the domains are narrow. On the other


Figure 3. Hard-elastic PP. CDFs $z\left(r_{12}, r_{3}\right)$ on a logarithmic scale as computed from SAXS data taken during a continuous straining experiment with an elongation at break $\varepsilon_{b}=2$.1. At $\varepsilon=1$, a layer system is observed. The length labeled "le" indicates double the average extension of the layers. At $\varepsilon=2$, the layer system is just vanishing. Labels " $\mathrm{fl}$ " and " $\mathrm{ft}$ " indicate average length and thickness of the remnant needle-shaped domains, 
hand, the ridges are long in straining direction. This means that the corresponding domains are long. We propose to call long and narrow domains needles. Thus, the ridges indicate the presence of needle-shaped domains. ${ }^{[52,53]}$ Only two ridges are visible. Thus, the distances among neighboring needles are not correlated. At $\varepsilon=1$, the layers are still predominant. The width of the respective reflections (label: "le") is related to their lateral extension.

At $\varepsilon=1.5$, the lamellae system is no longer prevailing. At $\varepsilon=2.0$, the last layers are vanishing. The nanostructure is made from uncorrelated needles. Thus, the material disrupts when all the lamellae have been consumed. The same result is reported from straining experiments of polyamide-6. ${ }^{[54]}$

Moreover, it appears noteworthy that the average thickness of the needles is constant $(8 \mathrm{~nm})$ during the experiment. This value is considerably higher than the experimental resolution $\left(1 / s_{\max }\right.$ with $\left.s_{\max }=0.18 \mathrm{~nm}^{-1}\right)$ of the SAXS setup. Therefore, we assume that this finding is no artifact, but an indication of a nanostructural feature. Because the needles do not exhibit transverse contraction, they should consist of rigid material. Such needles may be identified by the fibrillar crystallites as described by Noether. ${ }^{[33]}$

\section{Load-Cycling}

In the load-reversal experiment the elongation of the sample is oscillated in the elastic ${ }^{[33]}$ regime of the hardelastic material.

\section{The First-Cycle Scattering Patterns}

The lapse of the stress-strain curve and some of the recorded and combined SAXS/USAXS patterns are shown in Figure 4. Filled circles on the curve mark the average elongations and stresses related to the recorded scattering patterns. Numbers placed beside some of the circles indicate the serial number of the respective pattern. While a single pattern is exposed, stress and strain are continuously changing. The highlighted part of the curve around pattern 11 indicates the region that has been traversed during its exposure.

In Figure 4, the first seven scattering patterns are strung alongside the stress-strain curve. The unstrained material (pattern 0) exhibits on the meridian $\left(s_{3}\right)$ the typical twopoint diagram of a highly oriented lamellae ensemble. Even a strong second order of the long period peak is visible. The central scattering and the USAXS is moderate. Just after the beginning of the test (pattern 1) the USAXS in the center has considerably increased. The graphics had to be re-scaled, and therefore the first-order long-period peak looks weaker. In fact, only the second-order peaks have become weaker. The reflections appear blurred up to the beginning of the long linear region of the curve. The



Figure 4. Hard-elastic PP. Load-reversal mechanical test monitored by SAXS. Circular dots show where SAXS patterns have been recorded. Numerical labels indicate their sequence. The highlighted part of the curve near label 11 indicates the part of the curve traversed during the recording of pattern 11. The drawing direction with respect to the patterns, $s_{3}$, is indicated by a double-head arrow. The scattering patterns display the range $-0.15 \mathrm{~nm}^{-1}<s_{12}, s_{3}<0.15 \mathrm{~nm}^{-1}$.

scattering patterns are continuously changing, and exhibit several overlapping features. Thus, it appears inappropriate to directly interpret the patterns resorting to simplified structural notions.

\section{Fatigue: Variation of Scattering From Cycle to Cycle}

Mechanical fatigue of the material can be studied by following the curved arrows in Figure 4. The corresponding scattering patterns are shown in Figure 5. Again, direct interpretation of observed features does not return quantitative information on the evolution of the nanostructure made from crystalline and amorphous domains. The long-period peaks are too wide for a thorough determination of their position. Information on the lateral extension of the lamellae is hardly accessible. An exception is the scattering power that exhibits considerable oscillations. Therefore, the presentation shows patterns which are normalized to their highest intensity. For a quantitative analysis one may now try to extract the topological information of the two-phase structure from the scattering patterns, and to display it in real space as a CDF. ${ }^{[32]}$

\section{First Cycle: CDF Analysis}

The first four patterns recorded monitor the steep linear increase in the stress-strain curve (cf. Figure 4, patterns 03). The corresponding multidimensional $\mathrm{CDF}^{[30,32]}$ are presented in Figure 6.

The experiment has been reproduced three times with different samples. The unstrained material shows the typical CDF of a highly oriented ensemble of layers. Counted from the center, four peaks of nearly triangular 


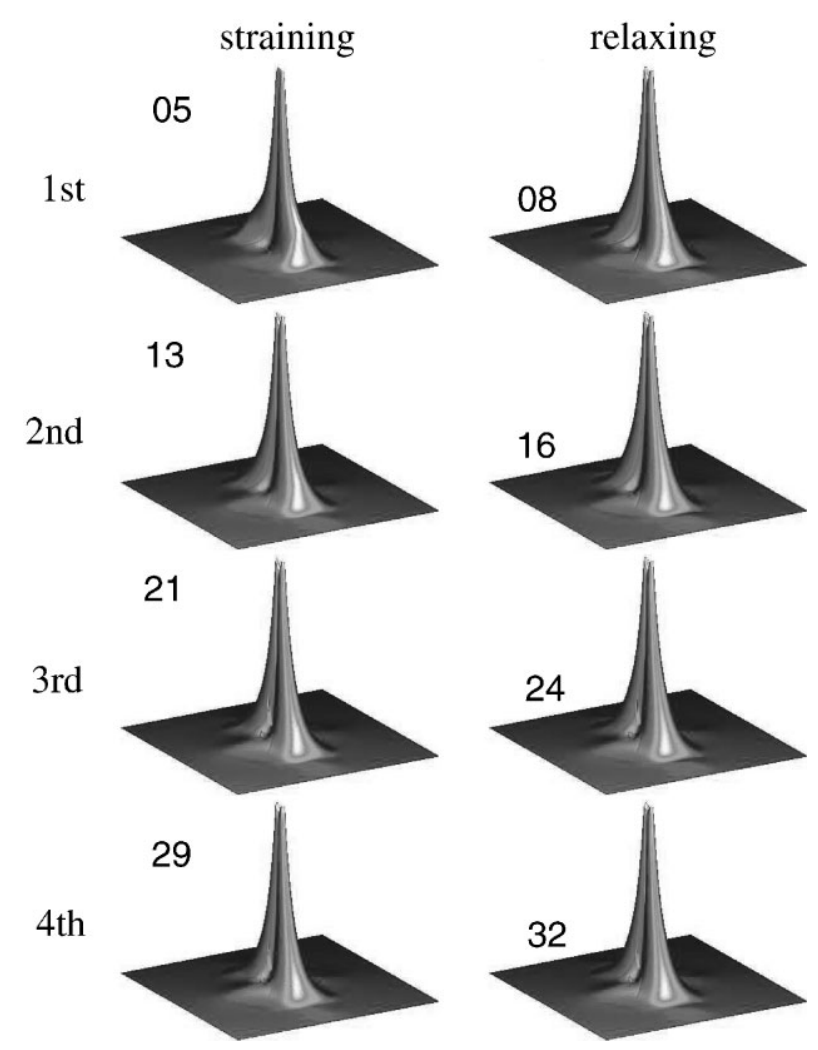

Figure 5. Load-reversal test of PP monitored by SAXS. Variation of the scattering pattern at constant machine elongation (cf. Fig. 4, label "fatigue") in four cycles under strain (left) and during relaxation (right), respectively. The patterns show the combined SAXS/USAXS intensity in the region $-0.1 \mathrm{~nm}^{-1}<s_{12}, s_{3}<0.1 \mathrm{~nm}^{-1}$. Numerical labels indicate the serial number of scattering patterns.

shape and decreasing height are observed. These peaks are generated at positions, which correspond to the average distances of layer surfaces from each other. Compared to other technical polymer materials, the range of order between the crystalline lamellae is quite high. The width of the broadest triangles in the direction of $r_{12}$ is related to the lateral extension of the lamellae. The distance between neighboring lamellae from each other is the long period $L$. An average value is given by the position of the first minimum of the $\mathrm{CDF}$ on the meridian, $r_{3}$ (cf. Figure 8). For the unstrained material, we determine $L=17.7 \mathrm{~nm}$.

At $\varepsilon=0.02$, we still find the same long period ( $L=$ $17.8 \mathrm{~nm}$ ). We now discover only three correlated layers in the stack. Noteworthy is the clear striation of the layer peaks, which is still present at $\varepsilon=0.06(L=18.8 \mathrm{~nm})$. At $\varepsilon=0.12(L=20.1 \mathrm{~nm})$, the yield point has been crossed, and the layer peaks are no longer striated.

Such a striation means that at least some of the layers must have been subjected to a density modulation in lateral direction. This modulation can either be attributed to the amorphous, or to the crystalline layers. Thus, either nanometer-size voids are torn open in some amorphous

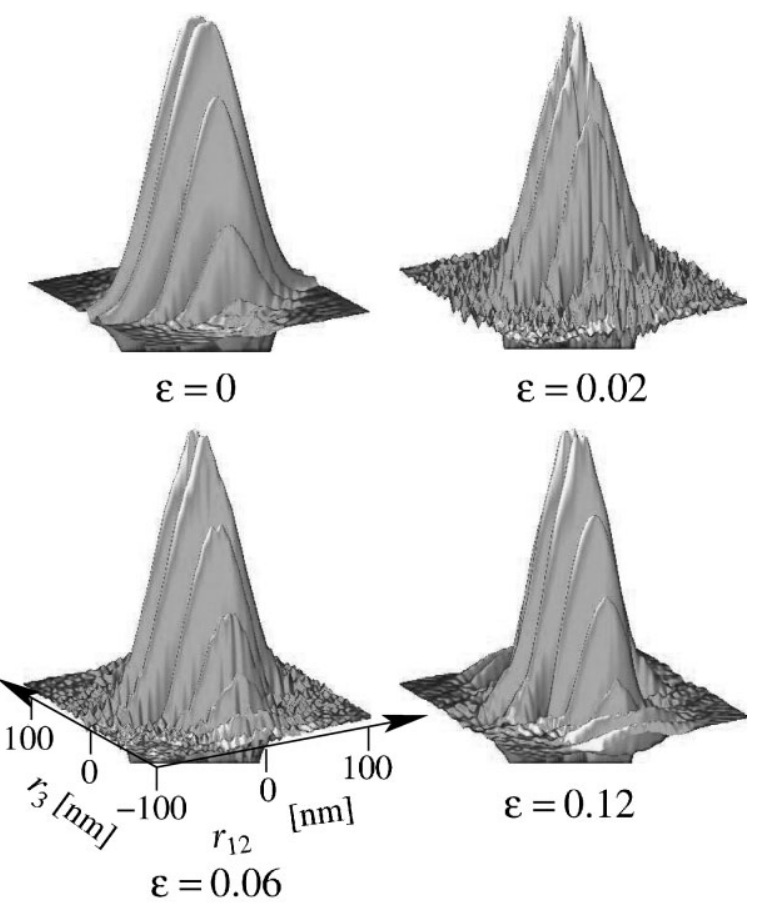

Figure 6. CDFs $z\left(r_{12}, r_{3}\right)$ on a logarithmic intensity scale showing the nanostructure evolution in highly oriented PP as a function of true elongation, $\varepsilon$. Combined SAXS/USAXS data recorded in timeresolved dynamic straining experiments.

layers, or some of the crystalline layers are breaking into blocks. Moreover, because after the yield point there are only smooth lamellae left over, the nanopores must have been merged into micropores, or the fragments of the weak lamellae must have molten. Figure 7 sketches the two possibilities. The generation of microporosity ${ }^{[38,39,44]}$
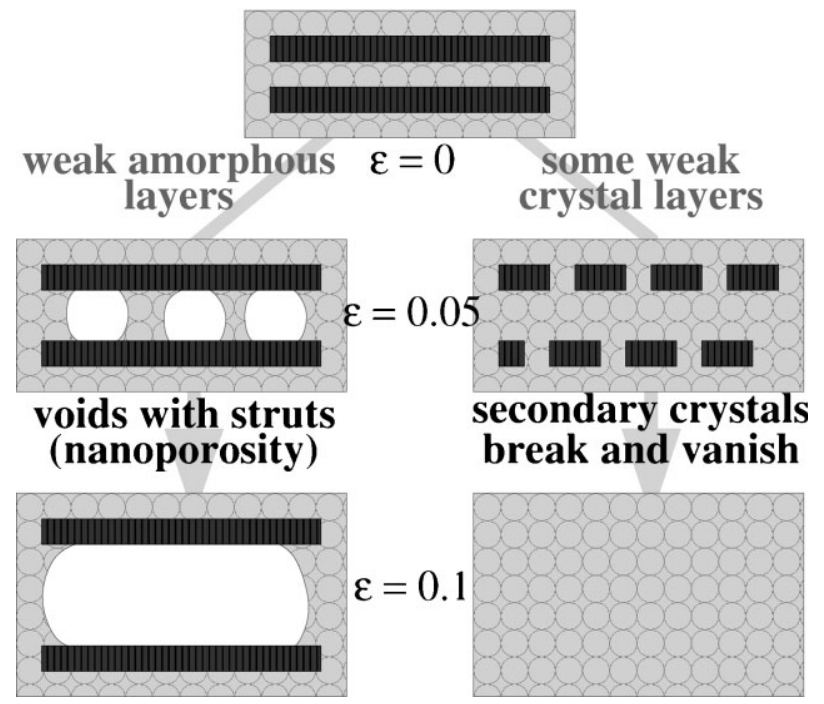

Figure 7. Two possible topological models for the explanation of the "blocky layers" observed in the scattering data of hard-elastic PP during straining. 


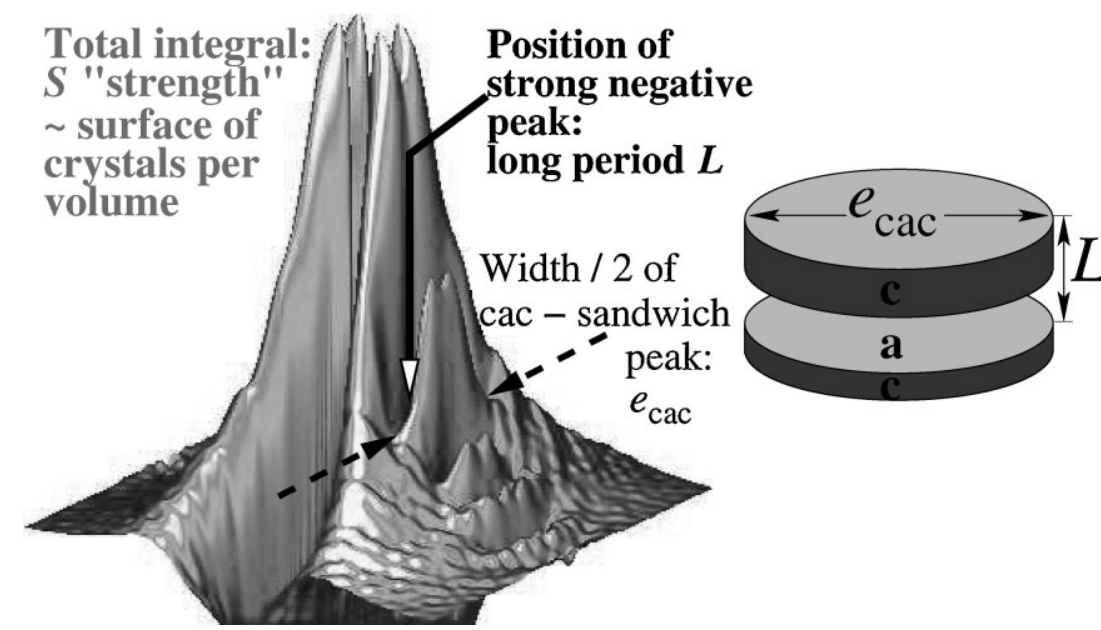

Figure 8. Simple methods of nanostructure parameter extraction from a CDF. The strength $S$ is a measure of surface between crystals and amorphous layers, $L$ is an average long period, and $e_{\mathrm{cac}}$ is an average lateral extension of the layer-shaped domains.

bridges between neighboring layers (cross-hatched structure ${ }^{[55]}$ ). Therefore, a determination requires peak separation, whereas $e_{\text {cac }}$ can be determined graphically. The phenomenon of bridge formation has been reported in a previous study on the nanostructure relaxation after stopping the extensometer. ${ }^{[43]}$

The strength is $S(\varepsilon=0)=30$ (in relative units) for the unstrained material. With increase in elongation it initially decreases [S $(\varepsilon=0.02)=15, \quad S(\varepsilon=0.06)=5]$, and begins to increase, again, after the yield point has been reached $[S(\varepsilon=0.12)=11, S$ $(\varepsilon=0.18)=28]$. From pattern 0 to pattern 4 the long period $L$ is continuously increasing $(L(\varepsilon=0)=18 \mathrm{~nm}, L(\varepsilon=0.18)=$ $23 \mathrm{~nm}$ ), but the lateral extension shows no increase $\left(e_{\text {cac }} \quad(\varepsilon=0)=30 \mathrm{~nm}, \quad e_{\text {cac }}\right.$ $(\varepsilon=0.18)=25 \mathrm{~nm})$. In particular the decrease in $S$ only permits to conclude that the initial striation of the layer

in hard-elastic thermoplastic material by straining is the basis of an important technical application of these polymers. Nevertheless, according to experience microporosity is only created, if the straining direction and the orientation direction of the material are different. ${ }^{[40]}$

If the observed dissection of lamellae peaks is a result of nanoporosity (left path in Figure 7), the integral $S=z\left(r_{12}, r_{3}\right) \mathrm{d} r_{12} \mathrm{~d} r_{3}$ must increase considerably, because it is proportional to the inner surface of the nanostructure multiplied by the square of the density jump at the phase boundary. This density jump becomes extreme upon formation of voids.

On the other hand, if the dissection is attributed to the failure of weak lamellae directly followed by melting of the fragments (right path in Figure 7), the disappearance reduces the surface, and the strength $S$ of the CDF signal decreases.

Not only the strength $S$, but also some other nanostructure parameters can easily be extracted from the CDF. The applied procedures are sketched in Figure 8. The average long period, $L$, is determined from the position of the minimum between the first and the second triangular peak. Half the base-length of the second triangular peak is a measure of the lateral extension, $e_{\mathrm{cac}}$, of a sandwich made from two layers of one kind which are separated by one layer of the other kind. Because we observe that the correlation among layers is low during load-cycling, $e_{\text {cac }}$ is, to a first approximation, the lateral extension of a sandwich made from two crystal lamellae. We have chosen this measure instead of measuring the extension of a single crystalline layer, because this feature is strongly interfering with a peak that indicates the formation of reflections in the CDF is related to a breakup of some (e.g., weak, secondary) crystal lamellae. Thus, the time-resolved experiment shows that the weak lamellae do not melt down from their borders, but disrupt into a mesostructure of blocks first, before they vanish. Moreover, the observed long-ranging striation shows that the blocks are arranged rather regularly in the plane of their "mother layer" similar to the pieces in a chocolate bar. This blocky mesostructure of PP has first been found and described by Strobl and coworkers ${ }^{[56]}$ in crystallization experiments of PP. Now our experiments indicate that even during mechanical loading of weak PP lamellae, this mesostate may be traversed. In conjunction with the notions developed for crystallization of lamellae from blocks, it appears suggesting that poorly welded lamellae are breaking into their constituents before the latter are melting. Moreover, such blocks have even been observed in an electron microscopic study of pre-stretched hard-elastic material ref. ${ }^{[40]}$, Figure 10a), when the direction of strain is the orientation direction of the material. However, the material studied is not PP but polyethylene. In excess of the results of electron microscopy, our in situ scattering experiment exhibits that (i) already at low elongation blocks are generated by breaking lamellae, (ii) the pieces are placed at regular intervals, (iii) the pieces are dynamically torn apart and vanish (melt). This observation of early block melting is in full agreement with the results of a deformation calorimeter study ${ }^{[57]}$ of hard-elastic PP. The authors report a clear partial melting effect for $0<\varepsilon<0.1$. According to the study, the melting effect is considerably reduced, if the material has been annealed at high temperature before straining. From the wavelength 


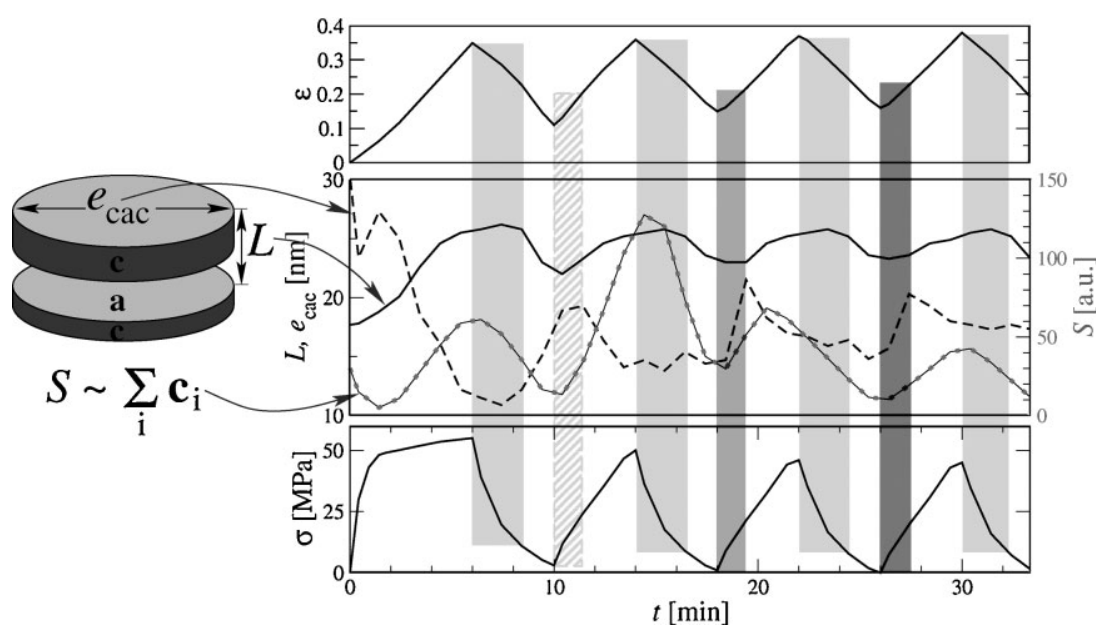

Figure 9. Dynamic load-reversal mechanical test of hard-elastic PP film at a strain rate of $\dot{\varepsilon} \approx 10^{-3} \mathrm{~s}^{-1}$. As a function of the elapsed time $t$ the macroscopic parameters elongation, $\varepsilon$ (top graph), and tensile stress, $\sigma$ (bottom graph) are displayed together with topological nanostructure parameters (middle). In the middle diagram the solid line shows the long period, $L$. The broken line displays the lateral extension, $e_{\mathrm{cac}}$, of a sandwich made from two crystalline lamellae. The line with circular dots exhibits the variation of the strength, $S$, of the CDF. Vertical bars indicate zones of strain-induced crystallization (dark gray) and relaxation-induced melting (light gray), respectively.

decreasing, whereas the decrease in $S$ is slowing down. Now the melting process is no longer predominant, and the contraction of the amorphous phase is reflected in the decrease in the average long period.

During the entire second straining phase $S(t)$ is increasing. Nevertheless, the process does not appear to be a simple one; in the beginning, the lateral extension of the lamellae remains high. This finding may be explained by moderate strain-induced crystallization (highlighted by a hatched dark gray box). To the right of this box the lateral extension $e_{\text {cac }}$ is decreasing. A possible explanation is a generation of surface $S$ by rupture of crystalline lamellae into stable fragments. The lateral extension of the fragments is low $(15 \mathrm{~nm})$ at an average long period of $25 \mathrm{~nm}$.

Let us now discuss the regions indicated by filled dark gray boxes in the third and the fourth cycle. They are

of lateral undulation on the layer peaks, we estimate an average distance between the blocks of $10 \mathrm{~nm}$ for $\varepsilon=0.02$. At $\varepsilon=0.12$, the amplitude of the undulation is much lower, and the wavelength of block arrangement has grown to $25 \mathrm{~nm}$.

Fatigue: $C D F$ and Nanostructure Evolution from Cycle to Cycle

In order to discuss the evolution, we have plotted the resulting nanostructure parameters together with the macroscopic mechanical data as a function of the elapsed time $t$. Figure 9 presents the result.

The top chart shows the true elongation, $\varepsilon(t)$, imposed to the material and measured at the point of X-ray irradiation. The four load-reversal cycles are easily identified. The chart in the middle reports the extracted nanostructure parameters, $L(t), e_{\mathrm{cac}}(t)$, and $S(t)$. The bottom diagram presents the macroscopic resistance, $\sigma(t)$, which the material is opposing to strain.

First, let us examine the regions in each strain cycle that are highlighted in light gray and start at the top dead center. During each of these intervals the long period, $L$, is almost constant at a high level. Simultaneously, in each cycle, $S(t)$, (crystallite surface, i.e., approximately the number of lamellae) is considerably decreasing, whereas the lateral extension of the layers is hardly changing. Altogether, this observation appears to be an indication for relaxation melting of crystalline lamellae. To the right of the light-gray boxes but still before the end of the relaxation half-cycle, the long period is considerably extending from the bottom dead center into the region of straining and end where the stress exceeds ${ }^{\mathrm{a}} \sigma(t)>20 \mathrm{MPa}$. In spite of the continuous straining, the long period remains on a low level, whereas the lateral extension of the crystallites is strongly increasing. An increase in $S$ is observed, as well. This is a clear indication of strain crystallization. If existing crystallite blocks would simply merge, the long period would have to increase and $S$ would decrease or stay constant.

However, the crystallization is not continued throughout the entire ascent of strain. After the end of the intervals marked in dark gray, the average lateral extension of the lamellae is suddenly decreasing. This observation can be explained by disruption of extended lamellae due to the applied high stress. The increasing long period supports the interpretation that crystallization has considerably slowed down. Thus, it appears convincing that in the middle of the straining branch a new nanostructure evolution mechanism is setting in, namely crystal disruption. The identification of such points in the load cycle appears to be important both for an understanding of the mechanical properties and for the design of models which are capable to predict mechanical properties of complex polymer materials.

\footnotetext{
${ }^{\text {a In a book chapter }}{ }^{[58]}$ some of the data have been presented in order to demonstrate the method. All the stress values given there are by $27 \mathrm{MPa}$ too high, because we had forgotten to subtract the basic load imposed by the weight of the upper cross-head.
} 

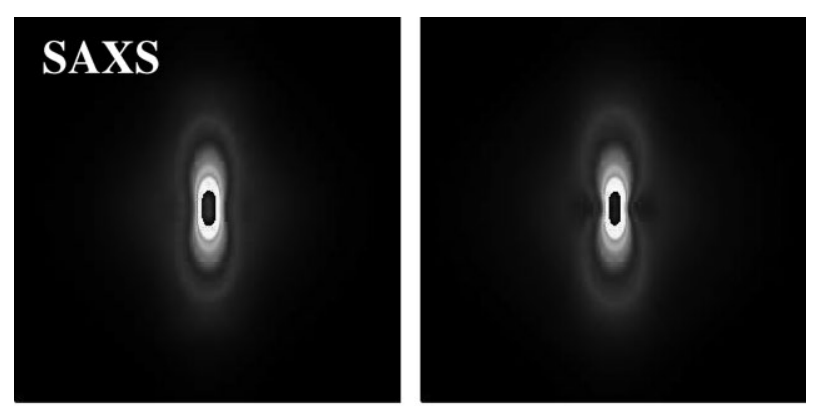

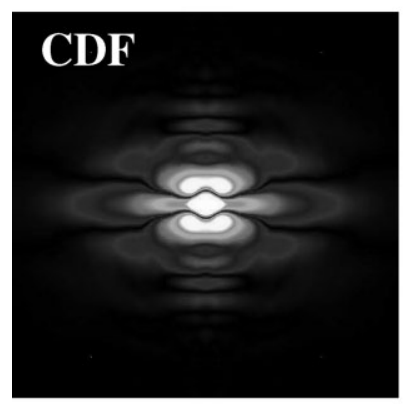

dynamic

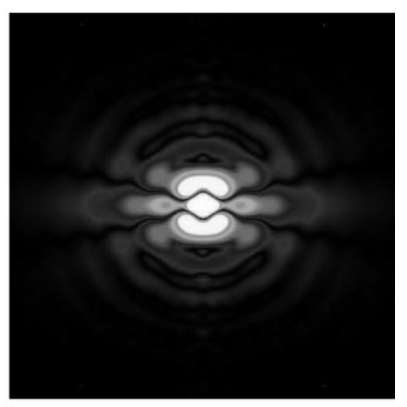

resting
Figure 10. Nanostructure relaxation during the first minute of rest after the load-cycling experiment at $\varepsilon=0.18$ on logarithmic scales. The SAXS patterns in the top represent a square of $-0.08 \mathrm{~nm}^{-1}<\mathrm{s}_{12}, \mathrm{~s}_{3}<0.08 \mathrm{~nm}^{-1}$ in identical scaling. The central dark spot is a result of intensity overflow. The bottom row shows $\left|z\left(r_{12}, r_{3}\right)\right|\left(-100 \mathrm{~nm}<r_{12}, r_{3}<100 \mathrm{~nm}\right)$. Straining direction $\left(s_{3}\right.$ and $r_{3}$, respectively) is vertical.

Finally, we turn to the structure variation in the first straining cycle between $2 \min <t<6 \min (\varepsilon: 0.1 \rightarrow 0.35)$, in which the stress, $\sigma(t)$, is only moderately increasing. Here, we observe a linear increase in $S$, accompanied by increasing long period and decreasing lateral extension of the crystallites. This finding may be interpreted as an indication for a superposition of strain crystallization and disruption of extended lamellae.

In summary, it appears most probable that crystallization, rupture of lamellae, and melting of fragments are continuously reshaping the domains of the PP material in the repeated cycles of a fatigue test. The long-period cycle exhibits a phase shift with respect to the imposed strain cycling. Moreover, there is an indication of amplitude attenuation. The curve $\sigma(t)$ is demonstrating the fatigue of the material by decreasing stress peaks.

\section{Structure Relaxation After the Load-Reversal Test}

As reported in a previous paper, ${ }^{[43]}$ the nanostructure information gathered by SAXS is different for PP, if the SAXS pattern is exposed while the extensometer is idle. Let us demonstrate the effect by investigating what happens after the load-cycle test is finished. Directly after the machine has been stopped, an additional scattering pattern has been recorded. The comparison is shown in pseudocolor in Figure 10. On the left side of the figure, i.e., while the machine has still been running, the CDF exhibits smooth streaks of an ensemble of extended lamellae. On the right, i.e., during the first minute after machine is stopped, the CDF additionally shows blocky domains. Moreover, the blocks appear arranged in the lateral plane. This is indicated by the regular modulation of the central streak. The finding can be explained by block crystallization which sets in as the extensometer is stopped.

\section{Mechanisms in the Stress-Strain Diagram}

A sketch in which the discussed mechanisms are projected onto the stress-strain diagram of the load-cycle experiment is presented in Figure 11.

For the relaxation branches of all the cycles our SAXS analysis is indicating the same kind of mechanism. Relaxation triggers the melting of crystalline domains, and this process is slowed down as the branch is traversed.

In the beginning of the strain branches it appears just the opposite: strain crystallization is widening existing lamellae and introducing new ones. As a limiting stress of $20 \mathrm{MPa}$ is exceeded, the slope of the strain branches becomes somewhat less steep and lamellae disruption starts. Nevertheless, in the second and fourth cycle the SAXS analysis tells that strain crystallization (now of blocky crystallites) remains dominant, whereas a lot of weak blocks appear to be molten in the third cycle. This is a strange observation and shows the limits of our analysis, although there is an indication in the stress-strain curve itself that is supporting our strange finding. Namely, the final slope of the strain branch (arrows with triangular filled double head) is less steep in the third cycle. Thus, as the SAXS analysis yields an indication that melting of weak blocks is predominant, the material appears to be

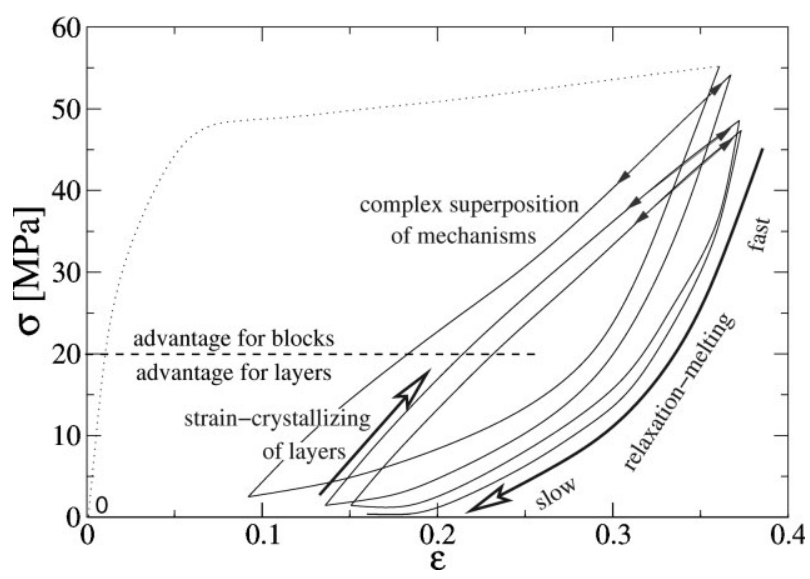

Figure 11. Mechanisms of nanostructure evolution in the stressstrain diagram of a slow load-cycling test of hard-elastic PP. 
softer than in those cases where crystallization of new blocks appears to dominate.

\section{Conclusion}

In our study, we have tested the possibility to monitor the evolution of nanostructure in a semicrystalline polymer material during a mechanical test by X-ray scattering. With some effort and for low strain rates this is already possible using presently available instrumentation. The experiment yields a description of the dynamic mechanisms, which are responsible for rupture or fatigue of the material.

Soon the time-resolution will become much better, when detectors based on the PILATUS principle ${ }^{[59]}$ will be available. Even the number of powerful synchrotron sources will soon be increasing. Then it will become possible to substantially increase the strain rate without loosing image quality, and, thus, to approach strain rates in the range of practical relevance.

Acknowledgements: We acknowledge HASYLAB, Hamburg, for provision of the synchrotron radiation facilities at beamlines A2 and BW4. Financial support by the European Commission under the seventh Framework Programme in the project NANOTOUGH is gratefully acknowledged.

Received: April 13, 2008; Revised: June 23, 2008; Accepted: June 30, 2008; DOI: 10.1002/macp.200800199

Keywords: fatigue analysis; mechanical properties; microstructure; poly(propylene) (PP); small-angle X-ray scattering (SAXS)

[1] D. J. Hughes, A. Mahendrasingam, C. Martin, W. B. Oatway, E. L. Heeley, S. J. Bingham, W. Fuller, Rev. Sci. Instrum. 1999, 70, 4051.

[2] M. F. Butler, A. M. Donald, W. Bras, G. R. Mant, G. E. Derbyshire, A. J. Ryan, Macromolecules 1995, 28, 6383.

[3] M. F. Butler, A. M. Donald, A. J. Ryan, Polymer 1997, 38, 5521.

[4] D. J. Hughes, A. Mahendrasingam, W. B. Oatway, E. L. Heeley, C. Martin, W. Fuller, Polymer 1997, 38, 6427.

[5] D. J. Blundell, A. Mahendrasingam, D. McKerron, A. Turner, R. Rule, R. J. Oldman, W. Fuller, Polymer 1994, 35, 3875.

[6] J. Wu, J. M. Schultz, F. Yeh, B. S. Hsiao, B. Chu, Macromolecules 2000, 33, 1765.

[7] M. Brandt, W. Ruland, Acta Polym. 1996, 47, 498.

[8] L.-Z. Liu, B. S. Hsiao, B. X. Fu, S. Ran, S. Toki, B. Chu, A. H. Tsou, P. K. Agarwal, Macromolecules 2003, 36, 1920.

[9] W. Wilke, M. Bratrich, J. Appl. Cryst. 1991, 24, 645.

[10] D. Grubb, V. Bala, Polym. Mater. Sci. Eng. 1999, 81, 357.

[11] B. X. Fu, B. S. Hsiao, S. Pagola, P. Stephens, H. White, M. Rafailovich, J. Sokolov, P. T. Mather, H. G. Jeon, S. Phillips, J. Lichtenhan, J. Schwab, Polymer 2000, 42, 599.
[12] F. Yeh, B. S. Hsiao, B. B. Sauer, S. Michel, H. W. Siesler, Macromolecules 2003, 36, 1940.

[13] N. Stribeck, S. Polizzi, P. Bösecke, H. G. Zachmann, Rev. Roum. Chem. 1989, 34, 635.

[14] N. Stribeck, P. Bösecke, S. Polizzi, Colloid Polym. Sci. 1989, 267, 687.

[15] N. Stribeck, J. Polym. Sci., Part B: Polym. Phys. 1999, 37, 975.

[16] N. Stribeck, S. Fakirov, D. Sapoundjieva, Macromolecules 1999, 32, 3368.

[17] S. Fakirov, O. Samokovlijsky, N. Stribeck, A. A. Apostolov, Z Denchev, D. Sapoundjieva, M. Evstatiev, A. Meyer, M. Stamm, Macromolecules 2001, 34, 3314.

[18] N. Stribeck, E. Buzdugan, P. Ghioca, S. Serban, R. Gehrke, Macromol. Chem. Phys. 2002, 203, 636.

[19] N. Stribeck, R. Androsch, S. S. Funari, Macromol. Chem. Phys. 2003, 204, 1202.

[20] N. Stribeck, S. Fakirov, A. A. Apostolov, Z. Denchev, R. Gehrke, Macromol. Chem. Phys. 2003, 204, 1000.

[21] N. Stribeck, S. S. Funari, J. Polym. Sci., Part B: Polym. Phys. 2003, 41, 1947.

[22] B. B. Sauer, R. S. McLean, D. J. Brill, D. J. Londono, J. Polym. Sci., Part B: Polym. Phys. 2002, 40, 1727.

[23] S. Murakami, M. Yamakawa, M. Tsuji, S. Kohjiya, Polymer 1996, 37, 3945.

[24] R. Séguéla, J. Prud'homme, Macromolecules 1988, 21, 635.

[25] H. S. Lee, S. R. Yoo, S. W. Seo, J. Polym. Sci., Part B: Polym. Phys. 1999, 37, 3233.

[26] G. E. Welsh, D. J. Blundell, A. H. Windle, J. Mater. Sci. 2000, 35, 5225.

[27] J. J. Hernández, M. C. García Gutiérrez, A. Nogales, D. R. Rueda A. Sanz, I. Sics, B. S. Hsiao, Z. Roslaniec, G. Broza, T. A. Ezquerra, Polymer 2007, 48, 3286.

[28] X. Chen, K. Yoon, C. Burger, I. Sics, D. Fang, B. S. Hsiao, B. Chu, Macromolecules 2005, 38, 3883.

[29] D. J. Blundell, G. Eeckhaut, W. Fuller, A. Mahendrasingam, C. Martin, J. Macromol. Sci. Phys. 2004, 43, 125.

[30] N. Stribeck, "X-Ray Scattering of Soft Matter", Springer, Heidelberg, New York 2007.

[31] P. Debye, H. Menke, "Ergebnisse der technischen Röntgenkunde", Vol. 2, J. Eggert, E. Schiebold, Eds., Akademische Verlagsgesellschaft, Leipzig 1931.

[32] N. Stribeck, J. Appl. Cryst. 2001, 34, 496.

[33] H. D. Noether, Int. J. Polymeric Mater. 1979, 7, 57.

[34] H. D. Noether, W. Whitney, Colloid Polym. Sci. 1973, 251 991.

[35] I. K. Park, H. D. Noether, Colloid Polym. Sci. 1975, 253, 824.

[36] S. Hild, W. Gutmannsbauer, R. Luthi, J. Fuhrmann, H.-J. Guntherodt, J. Polym. Sci., Part B: Polym. Phys. 1996, 34, 1953.

[37] J. Xu, M. Johnson, G. L. Wilkes, Polymer 2004, 45, 5327.

[38] M. B. Johnson, G. L. Wilkes, J. Appl. Polym. Sci. 2002, 83, 2095.

[39] M. B. Johnson, G. L. Wilkes, J. Appl. Polym. Sci. 2001, 81, 2944.

[40] G. L. Wilkes, H. Zhou, J. Mater. Sci. 1998, 33, 287.

[41] J. Bicerano, "Prediction of Polymer Properties", $3^{\text {rd }}$ edition, CRC Press, New York 2002.

[42] N. Stribeck, U. Nöchel, J. Appl. Cryst. 2008, accepted.

[43] N. Stribeck, U. Nöchel, S. S. Funari, T. Schubert, J. Polym. Sci. Polym. Phys. 2008, 46, 721.

[44] B. S. Sprague, J. Macromol. Sci. Phys. 1973, B8, 157

[45] N. Stribeck, A. Almendarez Camarillo, U. Nöchel, C. Schroer, M. Kuhlmann, S. V. Roth, R. Gehrke, R. K. Bayer, Macromol. Chem. Phys. 2006, 207, 1239.

[46] P. Debye, A. M. Bueche, J. Appl. Phys. 1949, 20, 518.

[47] G. Porod, Colloid Polym. Sci. 1951, 124, 83.

[48] C. G. Vonk, J. Appl. Cryst. 1973, 6, 81. 
[49] F. J. Baltá Calleja, C. G. Vonk, "X-Ray, Scattering of Synthetic Polymers”, Elsevier, Amsterdam 1989.

[50] W. Ruland, Colloid Polym. Sci. 1977, 255, 417.

[51] C. G. Vonk, Colloid Polym. Sci. 1979, 257, 1021.

[52] G. Porod, Fortschr. Hochpolym.-Forsch. 1961, 2, 363.

[53] W. O. Statton, Z. Kristallogr. 1968, 127, 229.

[54] N. S. Murthy, D. T. Grubb, J. Polym. Sci., Part B: Polym. Phys. 2002, 40, 691.

[55] D. R. Norton, A. Keller, Polymer 1985, 26, 704.
[56] T. Hugel, G. Strobl, R. Thomann, Acta Polym. 1999, 50, 214.

[57] D. Göritz, F. H. Müller, Colloid Polym. Sci. 1974, 252, 862.

[58] N. Stribeck, in: "Nano- and Micromechanics of Polymer Blends and Composites", Vol. 1, J. Karger-Kocsis, S. Fakirov, Eds., Hanser Publisher, München 2008.

[59] C. Broennimann, E. F. Eikenberry, B. Henrich, R. Horrisberger, G. Hülsen, E. Pohl, B. Schmitt, C. Schulze-Briese, M. Suzuki, T. Tomizaki, A. Toyokawa, A. Wagner, J. Synchrotron Rad. 2006, 13,120 